\title{
Impacto de las pruebas de bajo valor en pacientes ambulatorios de bajo riesgo
}

\author{
Impact of low-value testing on low-risk outpatients
}

\section{Comentado de:}

Bouck Z, et al. JAMA Intern Med. 2020;180(7):973-983. doi: 10.1001/jamainternmed.2020.1611. PMID: $32511668^{1}$

\section{Objetivo}

Evaluar la asociación de tres métodos de rastreo de bajo valor con la utilización posterior del sistema de salud y con desenlaces clínicos en pacientes ambulatorios de bajo riesgo que consultan para un chequeo anual de salud.

\section{Diseño y lugar}

Estudio de cohorte poblacional retrospectiva. Las fuentes de información empleadas fueron las bases de datos administrativas de atención médica de pacientes ambulatorios que realizaron un examen de salud anual entre abril de 2012 y marzo de 2016 en Ontario, Canadá.

\section{Participantes}

De las bases de datos mencionadas, se identificaron a las personas que cumplían al menos uno de los siguientes criterios de inclusión, generando tres cohortes de seguimiento:

- Cohorte 1. Pacientes adultos (18 años de edad o mayores) con bajo riesgo de enfermedad cardiovascular y pulmonar.

- Cohorte 2. Pacientes adultos con bajo riesgo de enfermedad cardiovascular.

- Cohorte 3. Mujeres (de 13 a 20 años o mayores de 69 años de edad) con bajo riesgo de cáncer de cuello uterino.

Se excluyeron a los pacientes que presentaran al menos una de las siguientes características: información demográfica incompleta, mayores de 105 años de edad, no residentes en Ontario, institucionalizados, o quienes fueron atendidos por un médico que hubiese realizado menos de 50 controles en la cohorte respectiva (es decir, que hubiese tenido menos de 50 oportunidades previas de haber solicitado los estudios en cuestión).

En las cohortes 1 y 2 además fueron excluidos los pacientes con antecedentes de enfermedad enfermedad cardiovascular y/o pulmonar, que hubiesen realizado consultas con especialistas (cardiólogo, neumólogo, cirujano torácico), con alto riesgo para enfermedad cardiovascular, o con enfermedades crónicas (enfermedad renal, HIV, etc.). En la cohorte 3 fueron excluidas las participantes con diagnóstico previo de cáncer ginecológico, antecedente de histerectomía, HIV o que estuviesen cursando un embarazo.

\section{Exposición}

En cada una de las cohortes, se evaluó la exposición a estudios de rastreo de bajo valor, definidos como:

- Cohorte 1. Una radiografía de tórax dentro de los siete días posteriores al control de salud.

- Cohorte 2. Un electrocardiograma (ECG) dentro de los 30 días posteriores al control de salud.

- Cohorte 3. Papanicolaou (PAP) dentro de los siete días posteriores al control de salud.

\section{Medición de los resultados principales}

Los resultados primarios evaluados fueron las visitas al especialista, las pruebas de diagnóstico y los procedimientos dentro de los 90 o 180 días posteriores a una prueba de bajo valor (si el paciente tuvo una radiografía de tórax, ECG o PAP) o al final de la ventana de observación de la exposición (si no se realizó la prueba).

Los resultados secundarios fueron la realización de procedimientos quirúrgicos, hospitalizaciones, consultas a la guardia o muertes en el año posterior al control de salud.

Dentro de cada cohorte, se estimó el puntaje de propensión de los participantes y se realizó un apareamiento 1:1 (expuestos vs. no expuestos). Los resultados fueron comparados mediante regresión logística multivariable.

\section{Resultados}

Luego del apareamiento, la cohorte 1 estuvo integrada por 43.532 pacientes (21.766 en cada grupo); la cohorte 2, por 245.686 pacientes (122.843 en cada grupo); y la cohorte 3, por 29.194 pacientes (14.597 en cada grupo).

La Tabla 1 resume los resultados principales, expresados como riesgo (el número de pacientes cada 100 individuos que realizaron la consulta o práctica en cuestión) y como diferencia de riesgo (el exceso de pacientes cada 100 individuos que realizaron la consulta o práctica en cuestión, en comparación con el grupo de referencia, los no expuestos a las prácticas de tamizaje).

Para los desenlaces resultados secundarios la probabilidad marginal fue baja en la mayoría de los eventos, de manera independiente de la exposición a las pruebas de tamizaje. En comparación con los pacientes no examinados, los pacientes que realizaron una radiografía de tórax tenían una mayor mortalidad al año (diferencia de riesgo [DR] 0,10; IC $95 \% 0,03$ a 1,81), mientras que las pacientes a quienes se les realizó un PAP tenían una menor mortalidad (DR -0,19; IC 95\% -0,32 a -0,06). No hubo diferencias en la mortalidad entre los pacientes que realizaron un ECG y quienes no lo hicieron. Tampoco hubo diferencias en las tasas de hospitalización entre los grupos de pacientes en las tres cohortes. Por último, los pacientes a quienes se les realizó una radiografía de tórax o un PAP tenían un mayor riesgo de tener al menos una visita al servicio de emergencias al año de seguimiento.

\section{Conclusiones}

Los pacientes de bajo riesgo que recibieron alguna de las pruebas de rastreo de bajo valor (radiografía de tórax, ECG o PAP) como parte de un examen anual de salud tenían más probabilidades de usar más servicios de atención médica (consultas a especialistas, pruebas diagnósticas y procedimientos) con posterioridad en comparación con pacientes no rastreados con estas pruebas.

Fuentes de financiamiento/Conflicto de interés de los autores: Uno de los autores informó haber recibido subsidios del Instituto Canadiense de Investigación en Salud y del Gobierno de Ontario, y recibir honorarios personales del Centro para la Práctica Efectiva, mientras otro de los autores informó haber recibido honorarios personales de Bind Insurance y ser miembro del comité directivo de Choosing Wisely International. No se informaron otros conflictos de interés.

Este estudio fue apoyado por ICES, que se financia con una subsidio anual del Ministerio de Salud y Atención a Largo Plazo de Ontario, Canadá (MOHLTC, por sus iniciales en inglés). Parte de este material se basa en datos e información recopilada y proporcionada por el MOHLTC de Ontario y el Instituto Canadiense de Información de salud. El estudio fue financiado por el Hospital Universitario de Mujeres. 
Tabla 1. Asociación entre las pruebas de rastreo de bajo valor y la realización posterior de consultas a especialistas, pruebas diagnósticas y procedimientos médicos. Notas: ECG: electrocardiograma; IC: intervalo de confianza; PAP: prueba de Papanicolaou.

\begin{tabular}{|c|c|c|c|c|c|}
\hline \multicolumn{2}{|c|}{ Desenlaces } & \multirow{2}{*}{$\begin{array}{c}\begin{array}{c}\text { Riesgo en } \\
\text { expuestos } \\
\text { (rastreados) } \\
(\%)\end{array} \\
1,39 \\
\end{array}$} & \multirow{2}{*}{$\begin{array}{c}\begin{array}{c}\text { Riesgo en no } \\
\text { expuestos (no } \\
\text { rastreados) } \\
(\%)\end{array} \\
0,52\end{array}$} & \multirow{2}{*}{$\begin{array}{l}\text { Diferencia de Riesgo } \\
\text { (DR) (IC } 95 \%) \\
0,87(0,69 \text { a } 1,05)\end{array}$} & \multirow{2}{*}{$\begin{array}{l}\text { Riesgo Relativo (IC } \\
95 \%)\end{array}$} \\
\hline & Consulta a neumonología & & & & \\
\hline $\begin{array}{l}\text { Radiografía de tórax } \\
\text { (90 días de }\end{array}$ & Broncoscopía & 0,16 & 0,02 & $0,14(0,08$ a 0,19$)$ & $8,50(3,02$ a 23,95$)$ \\
\hline seguimiento) & TC abdominal o torácica & 2,88 & 0,92 & $1,96(1,71$ a 2,22$)$ & $3,14(2,67$ a 3,67$)$ \\
\hline \multirow{4}{*}{$\begin{array}{l}\text { Cohorte 2: ECG } \\
\text { (90 días de } \\
\text { seguimiento) }\end{array}$} & Consulta a cardiología & 2,70 & 0,77 & $1,92(1,82$ a 2,02$)$ & $3,48(3,24$ a 3,74$)$ \\
\hline & Ecocardiograma transtorácico & 7,12 & 1,64 & $5,49(5,33$ a 5,65$)$ & $4,35(4,15$ a 4,57$)$ \\
\hline & Prueba de esfuerzo & 5,84 & 1,38 & $4,46(4,31$ a 4,61$)$ & $4,24(4,02$ a 4,46$)$ \\
\hline & Cateterismo cardíaco & 0,24 & 0,09 & $0,15(0,12$ a 0,18$)$ & $2,64(2,12$ a 3,28$)$ \\
\hline \multirow{3}{*}{$\begin{array}{l}\text { Cohorte 3: PAP } \\
\text { (180 días de } \\
\text { seguimiento) }\end{array}$} & Consulta a ginecología & 5,07 & 3,76 & $1,31(0,84$ a 1,78$)$ & $1,35(1,21$ a 1,50$)$ \\
\hline & Nuevo PAP & 56,22 & 3,44 & $52,78(51,92$ a 53,63$)$ & $16,35(14,98$ a 17,83$)$ \\
\hline & Colposcopía & 1,03 & 0,19 & $0,84(0,66$ a 1,01$)$ & $5,36(3,58$ a 8,02$)$ \\
\hline
\end{tabular}

\section{Comentario}

Sabemos que no es suficiente considerar si una práctica es beneficiosa para un paciente al momento de solicitarla, sino que también debemos valorar la seguridad y el balance de daños en relación a los beneficios esperados, incluyendo las preferencias del paciente. Esto recobra mayor importancia en las prácticas preventivas realizadas en pacientes sanos, en quienes resulta de vital importancia evaluar si el beneficio de la intervención médica justifica el daño potencial (por sobrediagnóstico, falsos positivos, etc.).

Este estudio analizó la cascada diagnóstica desencadenada por la realización de tres pruebas de rastreo realizadas en pacientes sin indicación, debido a la baja prevalencia de enfermedades entre poblaciones jóvenes y sanas, y a los riesgos asociados con estos exámenes de detección (de ahí su denominación de pruebas de bajo valor) ${ }^{2-4}$. Si bien una de las limitaciones del estudio es, precisamente, no poder garantizar que estas pruebas hayan sido solicitadas con fines de rastreo y no, por ej., por un hallazgo clínico no documentado, cuando se tienen en cuenta los criterios de exclusión utilizados resulta razonable asumir que en la mayoría de los pacientes incluidos no ha sido éste el caso. Sus hallazgos refuerzan la evidencia respecto al escaso beneficio que presentan las pruebas evaluadas frente a las consecuencias que pueden acarrear tanto para el paciente como para el sistema de salud. Cabe destacar que este estudio, realizado con datos recolectados de forma sistemática en Canadá, obliga además a reflexionar sobre la importancia de contar con registros de salud adecuados para realizar este tipo de análisis.

Hace falta mayor difusión de la información ya conocida en relación al daño potencial de las prácticas preventivas. Sin dejar de entender que los medios de comunicación cumplen un rol esencial, también resulta cierto que los mensajes en salud que más se reproducen en ese ámbito suelen enfocarse en "hacer", "más es mejor", y "prevenir es siempre mejor que curar". La educación de la población en relación a sus derechos y el involucramiento de los pacientes de manera activa en las decisiones sobre su salud requieren la provisión de información veraz y equilibrada tanto sobre los beneficios como sobre los daños.

Por último, los profesionales de la salud no quedamos exentos de esta tendencia. Instruir y enseñar en contexto de la prevención cuaternaria desde las carreras de grado resulta fundamental en estos tiempos en que las pruebas de bajo valor son sobreutilizadas. Los profesionales ya en actividad no sólo necesitan conocer las diferentes herramientas y recomendaciones a la hora de prescribir estudios diagnósticos y/o tratamientos, sino también comprender la lógica que sustenta las recomendaciones. Con este propósito se desarrolló en Argentina la "iniciativa interespecialidades" 5 , en la que diferentes asociaciones científicas generan recomendaciones de prácticas de salud, basándose en evidencia actualizada y buscando la protección de los pacientes. Una lógica similar representa la iniciativa "Choosing Wisely" 6 , cuya misión es promover las conversaciones entre los pacientes y sus médicos de manera que se brinden los cuidados médicos respaldados por evidencia, no redundantes, que minimicen el daño potencial y que sean realmente necesarios.

\section{Conclusiones de la comentadora}

Basándonos en la evidencia previa y en la aportada por este estudio, no resulta recomendable solicitar radiografiás de tórax, ECG o PAP a personas asintomáticas con bajo riesgo con fines de rastreo y por fuera de las recomendaciones vigentes. 
Catalina Morán [ Servicio de Medicina Comunitaria, Hospital Italiano de Buenos Aires. catalina.moran@hospitalitaliano.org.ar ]

Morán C Impacto de las pruebas de bajo valor en pacientes ambulatorios de bajo riesgo. Evid Actual Pract Ambul. 2021;24(3):e002143. Available from: https://dx.doi.org/10.51987/EVIDENCIA.V24I4.6966. Comentado de: Bouck Z, et al. Association of Low-Value Testing With Subsequent Health Care Use and Clinical Outcomes Among Low-risk Primary Care Outpatients Undergoing an Annual Health Examination. JAMA Intern Med. $2020 ; 180(7): 973-983$. PMID: 32511668

\section{Referencias}

1. Bouck Z, Calzavara AJ, Ivers NM, et al. Association of Low-Value Testing With Subsequent Health Care Use and Clinical Outcomes Among Low-risk Primary Care Outpatients Undergoing an Annual Health Examination. JAMA intern med. 2020;180(7):973-83. Available from: 10.1001/ jamainternmed.2020.1611.

2. Sacha-Bhatia R, Bouck Z, Ivers NM, et al. Electrocardiograms in Low-Risk Patients Undergoing an Annual Health Examination. JAMA Intern Med. 2017;177(9):1326-1333. Available from: 10.1001/jamainternmed.2017.2649.

3. Cervical Cancer: Screening; 2018. Available from: https://www.uspreventiveservicestaskforce.org/uspstf/recommendation/cervical-cancer-screening [Last access: 2021-08-05].

4. Choosing Wisely. Chest X-rays Before Surgery;. Available from: https://www.choosingwisely.org/patient-resources/chest-x-rays-before-surgery/ [Last access: 2021-08-05].

5. Iniciativa inter-especialidades;. Available from: http://interespecialidades.org/quienes-somos [Last access: 2021-08-05]

6. Choosing Wisely;. Available from: https://www.choosingwisely.org/ [Last access: 2021-08-05]. 